\title{
Optimal Reserve Allocation to Maximize Kinetic Energy in a Wind Power Plant
}

\author{
Gihwan Yoon*, Hyewon Lee*, Jinsik Lee*, Gi-Gab Yoon**, Jong Keun Park*** \\ and Yong Cheol Kang ${ }^{\dagger}$
}

\begin{abstract}
Modern wind generators (WGs) are forced or encouraged to participate in frequency control in the form of inertial and/or primary control to improve the frequency stability of power systems. To participate in primary control, WGs should perform deloaded operation that maintains reserve power using speed and/or pitch-angle control. This paper proposes an optimization formulation that allocates the required reserve to WGs to maximize the kinetic energy (KE) stored in a wind power plant (WPP). The proposed optimization formulation considers the rotor speed margin of each WG to the maximum speed limit, which is different from each other because of the wake effects in a WPP. As a result, the proposed formulation allows a WG with a lower rotor speed to retain more KE in the WPP. The performance of the proposed formulation was investigated in a 100-MW WPP consisting of 20 units of 5-MW permanent magnet synchronous generators using an EMTP-RV simulator. The results show that the proposed formulation retains the maximum amount of KE with the same reserve and successfully increases the frequency nadir in a power system by releasing the stored KE in a WPP in the case of a disturbance.
\end{abstract}

Keywords: Deloaded operation, Frequency support, Kinetic energy, Primary control, Wake effect, Wind power plant.

\section{Introduction}

Global wind power generation has increased signifycantly in recent decades because of technical advances. The global installed capacity of wind generation was 317 GW in 2013 and is expected to increase to $596 \mathrm{GW}$ by 2018 [1]. In Korea, the installed capacity of wind generators (WGs) was approximately $605 \mathrm{MW}$ in 2014 and will increase to $17 \mathrm{GW}$ by 2027 [2].

Variable-speed WGs are widely used for their maximum power point tracking (MPPT) control, which optimizes the rotor speed according to wind speed. However, WGs do not respond to changes in frequency; thus, the frequency stability for a highly-wind-penetrated power system is often degraded as a result of MPPT operation [3].

To solve this problem, frequency control requirements have been included in the grid codes $[4,5]$. These requirements can be classified into two groups: inertial and primary controls $[6,7]$. The former temporarily releases the kinetic energy (KE) stored in a rotating mass of WGs,

$\dagger$ Corresponding Author: Dept. of Electrical Engineering, WeGAT Research Center, and Smart Grid Research Center, Chonbuk National University, Korea. (yckang@jbnu.ac.kr)

* Dept. of Electrical Engineering and WeGAT Research Center, Chonbuk National University, Korea. (\{kihwan01, hyewonlee, jinsiklee\}@jbnu.ac.kr).

** Korea Electric Power Research Institute, Korea. (90102630@ kepco.co.kr)

*** Dept. of Electrical Engineering, Seoul National University, Korea (parkjk@snu.ac.kr)

Received: February 24, 2015; Accepted: June 5, 2015 whereas the latter uses the reserve that is retained by pitch and speed controls.

To emulate inertial control, WGs use auxiliary loops based on the rate of change of frequency and / or the frequency deviation. The inertial control capability of the WGs is dependent upon the stored KE [8]. To execute primary control, the WGs retain the reserve, which is obtained by pitching and / or overspeeding $[9,10]$. Overspeeding can increase the KE, while pitching cannot.

Methods have been proposed for retaining the required reserve in a WG [11] or wind power plant (WPP) [12] to provide primary frequency control. An optimization method for maximizing the KE in a WG was proposed to retain the required reserve [11]. In this optimization, the $\mathrm{KE}$ is maximized by retaining the reserve in the form of rotor speed, as opposed to maintaining the pitch angle. An algorithm that dynamically allocates the required reserve of a WPP to a WG proportional to its available power has been proposed [12]. The allocation algorithm considers wake effect in a WPP and thus allows a WG with a high wind speed to retain more reserve.

This paper proposes an optimization formulation that allocates the required reserve to the WGs to maximize the KE stored in a WPP. It assumes that a WPP should retain the required reserve to support frequency control. The proposed optimization formulation allows a WG with a low rotor speed to retain sufficient KE to maximize the KE of the WPP. In addition, the wind speed approaching a WG in a WPP is calculated with consideration of the wake effect. 
The performance of the proposed optimization formulation is investigated for a model system that consists of five synchronous generators (SGs) and a 100-MW WPP with 20 units of 5-MW permanent magnet synchronous generators (PMSGs) using an EMTP-RV simulator under various wind conditions.

\section{Optimal Reserve Allocation to Maximize the KE of a WPP Considering the Wake Effect}

The wind speeds arriving at the WGs in a WPP will vary because of the wake effect; thus, individual WGs will operate at different rotor speeds. This means that WGs have different margins between the operating rotor speed and the maximum rotor speed limit.

This paper aims to provide an optimization formulation that allocates the required reserve of a WPP to the WGs to obtain the maximum KE, which will be released to support frequency stability during a disturbance. We explain how a WG obtains the reserve, describe the wake effect model used in this paper, and formulate the optimization problem.

\subsection{PMSG model}

This subsection describes the overall features of the PMSG model used in this paper: a turbine model, twomass shaft model, and a PMSG controller. Fig. 1 shows a typical configuration of the PMSG model used in this paper.

The mechanical input power of the PMSG extracted from the wind, $P_{m}$, can be represented by

$$
P_{m}=\frac{1}{2} \rho \pi R^{2} v^{3} c_{P}(\lambda, \beta)
$$

where $\rho, R, v, \lambda$, and $\beta$ are the air density, blade length, wind speed, tip speed ratio, and pitch angle, respectively; in this paper, $\rho$ and $R$ are set to $1.225 \mathrm{~kg} / \mathrm{m}^{3}$ and $60 \mathrm{~m}$, respectively. $c_{P}$ is the power coefficient and can be represented by

$$
c_{P}(\lambda, \beta)=c_{1}\left\{c_{2} \lambda+\frac{c_{3}-c_{4}(2.5+\beta)+c_{5} \lambda}{e^{c_{6} \lambda_{i}}}\right\}
$$

where

$$
\begin{gathered}
\lambda_{i}=\frac{1}{\lambda+c_{7}(2.5+\beta)}-\frac{c_{8}}{c_{9}+(2.5+\beta)^{3}} \\
\lambda=\frac{\omega R}{v}
\end{gathered}
$$

and $c_{1}, c_{2}, c_{3}, c_{4}, c_{5}, c_{6}, c_{7}, c_{8}$, and $c_{9}$ are set to 0.645 , $0.00912,-5,0.4,116,21,0.08,0.035$, and 1 , respectively; in this paper, the maximum $c_{P}$ is set to 0.5 and $\omega$ is the

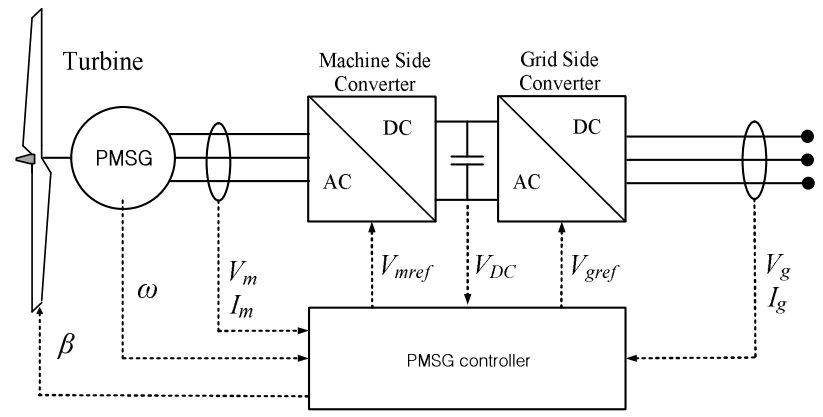

$V_{m}, I_{m}$ : voltage and current at MSC, $V_{g}, I_{g}$ : voltage and current at GSC, $V_{\text {mref }}$ : reference MSC voltage, $V_{\text {gref }}$ : reference GSC voltage, $V_{D C}$ : DC-link voltage $\omega$ : rotor speed $\beta$ : pitch angle

Fig 1. Typical configuration of a PMSG

rotor speed.

A two-mass model for simulating the mechanical dynamics of a wind turbine is represented by

$$
J \frac{d \omega}{d t}=T_{m}-K_{r} \omega-T_{e}
$$

where $J, K_{r}, T_{m}$, and $T_{e}$ are the moment of inertia, rotor damping coefficient, mechanical torque, and electromagnetic torque, respectively.

A PMSG controller regulates the machine-side converter (MSC) and grid-side converter (GSC) using the measured the voltages and currents at both sides of the converters and the rotor speed. In this paper, the MSC controls the torque of the PMSG, whereas the GSC maintains the DClink voltage and reactive powers injected into the grid. In addition, a pitch-angle controller is used to prevent the rotor speed from exceeding the maximum operating limit.

\subsection{Obtaining the reserve of the PMSG}

To support frequency control, a WG should retain the required reserve. To maintain the reserve, the power coefficient of a WG should be intentionally reduced by increasing the rotor speed and/or pitch angle.

Fig. 2 illustrates the power-speed curves of the PMSG. The black solid and dotted lines are the mechanical input power curves of the PMSG in p.u. under various wind speeds at the pitch angles of zero and four degrees, respectively; the blue solid line is the electrical output curve for MPPT operation, and the red dotted line indicates the maximum rotor speed limit of 1.1 p.u. The base values of power and rotor speed are set to $5 \mathrm{MW}$ and $94.2 \mathrm{rad} / \mathrm{s}$, respectively. Assuming that the PMSG is performing MPPT control at the wind speed of $9 \mathrm{~m} / \mathrm{s}$, the PMSG operates under conditions represented by the red circle in Fig. 2. The reserve can be obtained by overspeeding and/or pitching, during which the operating point should be moved from the red circle. Note that, unlike pitching, overspeeding can retain more $\mathrm{KE}$ in the rotor while 


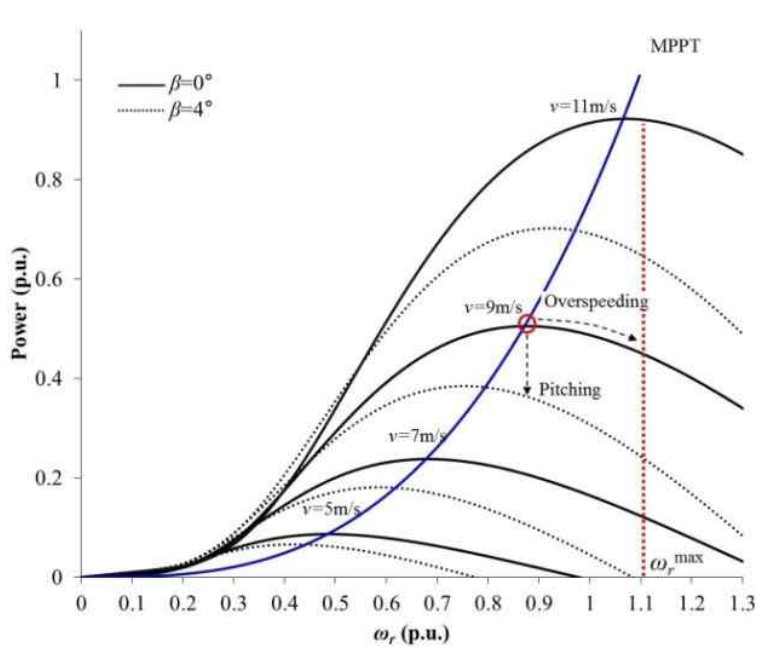

Fig. 2. Power- $\omega_{r}$ curves of the PMSG in different wind speeds and pitch angles

retaining the reserve.

\subsection{Wake effect}

In a WPP consisting of multiple WGs, the wind speed of the downstream WGs is reduced because of the upstream WGs; this kind of shadowing is referred to as the wake effect, which leads to differences between incoming wind speeds and corresponding power curves in WGs. Consequently, the WGs in a WPP operate at different conditions in terms of rotor speed and the chosen margin of the rotor speed limit.

\subsection{Optimization formulation to maximize $\mathrm{KE}$ in a WPP}

There are various combinations to retain the required reserve in a WPP. In this paper, the reserve of WGs is optimized to maximize the KE in a WPP to improve the frequency support capability. The KE stored in the rotating mass of a PMSG can be represented by

$$
\mathrm{KE}=\frac{1}{2} J \omega^{2}
$$

Normalizing both sides of (6) by the apparent power yields

$$
\mathrm{KE}_{\text {p.u. }}=H \omega_{r}^{2}
$$

where $H$ and $\omega_{r}$ are the inertia time constant of a PMSG in second and rotor speed in p.u., respectively.

The proposed optimization formulation is given by

$$
\operatorname{Max}\left\{\sum_{i=1}^{n} H_{i} \omega_{r, i}^{2}\right\}
$$

$$
\begin{gathered}
\text { subject to } \quad \omega_{r, i}^{o p t} \leq \omega_{r, i} \leq \omega_{r, i}^{\max } \quad i=1,2, \cdots, n \\
0 \leq \beta_{i} \leq \beta_{i}^{\max } \quad i=1,2, \cdots, n \\
P_{W P P}^{\max }(1-R E S)=\sum_{i=1}^{n} \frac{1}{2} \rho \pi R_{i}^{2} v_{i}^{3} c_{P, i}
\end{gathered}
$$

where $H_{i}$ is the normalized inertia time constant of the $i$-th PMSG, $\omega_{r, i}$ is the rotor speed of the $i$-th PMSG in p.u., $n$ is the number of PMSGs in a WPP. $\omega_{r, i}{ }^{o p t}$ and $\omega_{r, i}{ }^{\max }$ are the optimum rotor speed for MPPT control and the maximum rotor speed of the $i$-th PMSG. $\beta_{i}^{\text {max }}$ is the maximum pitch angle of the $i$-th PMSG. RES is the ratio of the reserve to the available power of the WPP, $P_{W P P}^{\max }$, which can be expressed as

$$
P_{W P P}^{\max }=\sum_{i=1}^{n} \frac{1}{2} \rho \pi R_{i}^{2} v_{i}^{3} c_{P, i}^{\max }
$$

The proposed optimization formulation maximizes the sum of KEs stored in PMSGs subject to the operating ranges of the rotor speed and pitch angle, and the required output of a WPP considering the reserve. Note that in the proposed optimization formulation the objective function is quadratic and a constraint related to $c_{P}$ is nonlinear.

There can be many ways to find a solution of the proposed optimization problem; however, it is beyond the scope of this paper. To find a solution of the proposed formulation, for convenience, this paper uses the sequential quadratic programming, because it is known as one of the algorithms suitable for solving nonlinearly constrained optimization problems [13].

\section{Model System}

Fig. 3 shows a model system chosen to investigate the performance of the proposed optimization. This system consists of five conventional SGs, a 100-MW PMSG-based WPP, and a static load of $600 \mathrm{MW}$ and 9 MVAr. Further information on the generators is presented in the following subsections.

\subsection{SGs}

The total generation capacity of five SGs in the model system is 800 MVA, which consists of two 200-MVA SGs, two 150-MVA SGs, and one 100-MVA SG. For convenience, it is assumed that all SGs are steam turbine generators, and the droop gain of the SGs is set to $5 \%$, which is the typical value.

\subsection{PMSG-based WPP}

The WPP consists of 20 units of a 5-MW PMSG. The distance between adjacent PMSGs is $840 \mathrm{~m}$, which is $7 D$, where $D$ is the diameter of the swept area of a PMSG. As 
shown in Fig. 3, three feeders are connected to a collector bus. The first and second feeders are composed of seven PMSGs, and the third feeder is composed of six PMSGs. The collector bus is connected to a 22-km-long submarine intertie cable through two 60-MVA substation transformers. The rated voltage of the inner and outer grids is $33 \mathrm{kV}$ and $154 \mathrm{kV}$, respectively.

Fig. 4 shows the power curve of the 5-MW PMSG, whose cut-in, rated, and cut-out wind speeds are $3 \mathrm{~m} / \mathrm{s}$, $11.3 \mathrm{~m} / \mathrm{s}$, and $25 \mathrm{~m} / \mathrm{s}$, respectively, and $\omega_{r, i}{ }^{\max }$ and $\beta_{i}{ }^{\max }$ are set to 1.1 p.u. and $30^{\circ}$, respectively.

The performance of the proposed formulation was investigated under various wind conditions using an active power control scheme for a disturbance, during which the frequency decreases to less than $59.98 \mathrm{~Hz}$. Fig. 5 shows the active power-control loop implemented in the PMSG, where $P_{M P P T}$ is the power reference for MPPT operation. All PMSGs used in this study are assumed to perform the deloaded operation prior to a disturbance. If a disturbance occurs and the system frequency drops below $59.98 \mathrm{~Hz}$, the active power reference is switched from $P_{W G, i}^{c m d}$ to $P_{M P P T}$ to

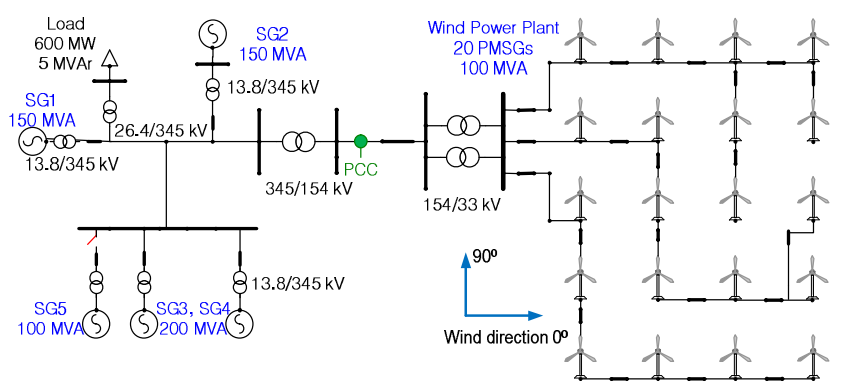

Fig. 3. Model system

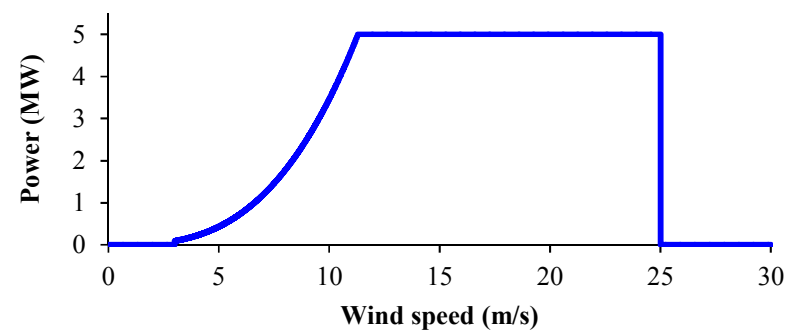

Fig. 4. Power curve of the PMSG

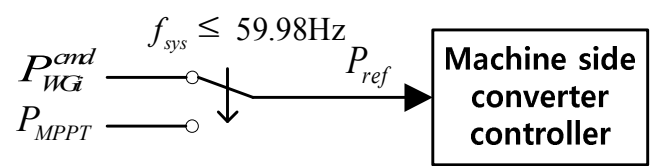

Fig. 5. Active power control scheme of the PMSG

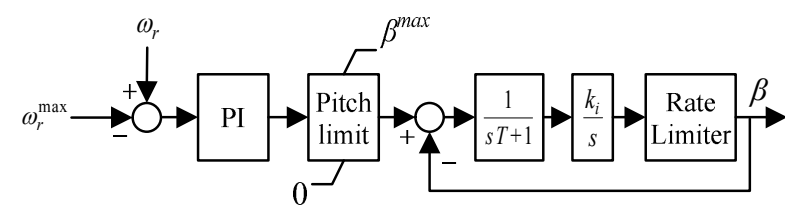

Fig. 6. Pitch control loop of the PMSG
Table 1. Wind speeds of PMSGs for three cases

\begin{tabular}{c|c|c|c||c|c|c|c||c|c|c|c}
\hline \multicolumn{3}{l||}{ Wind Speeds for Case } & \multicolumn{3}{c||}{ Wind Speeds for Case } & \multicolumn{3}{c}{ Wind Speeds for Case } \\
\multicolumn{3}{c|}{$1(\mathrm{~m} / \mathrm{s})$} & \multicolumn{3}{c||}{$2(\mathrm{~m} / \mathrm{s})$} & \multicolumn{3}{c}{$3(\mathrm{~m})$} \\
\hline 10 & 8.1 & 7.8 & 7.7 & 11 & 8.9 & 8.6 & 8.5 & 8.4 & 8.4 & 8.4 & 8.4 \\
\hline 10 & 8.1 & 7.8 & 7.7 & 11 & 8.9 & 8.6 & 8.5 & 8.5 & 8.5 & 8.5 & 8.5 \\
\hline 10 & 8.1 & 7.8 & 7.7 & 11 & 8.9 & 8.6 & 8.5 & 8.6 & 8.6 & 8.6 & 8.6 \\
\hline 10 & 8.1 & 7.8 & 7.7 & 11 & 8.9 & 8.6 & 8.5 & 8.9 & 8.9 & 8.9 & 8.9 \\
\hline 10 & 8.1 & 7.8 & 7.7 & 11 & 8.9 & 8.6 & 8.5 & 11 & 11 & 11 & 11 \\
\hline
\end{tabular}

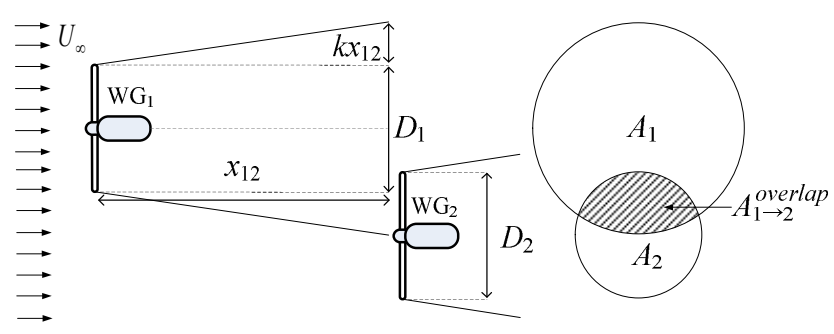

Fig. 7. Shadow cones in the Park model

release the stored KE and reserve power in the WPP. The pitch controller in the PMSG is used to restrict $\omega_{r, i}$ to $\omega_{r, i}{ }^{\max }$, as shown in Fig. 6, where $T$ is the time constant of the pitch servo controller and was set to $1 \mathrm{~s}$; the maximum rate limit was set to $10 \%$ s.

\subsection{Wake model}

The wind speeds arriving at the WGs are calculated using the Park model, which is based on Jensen's wake model and considers the cumulative impact of multiple shadowing and the wind direction [14]. Fig. 7 shows a shadow cone from an upstream WG. In the figure, $U_{\infty}$ is the incoming wind speed of the $\mathrm{WG}_{1}$ without any shadowing, $x_{12}$ is the radial distance between $\mathrm{WG}_{1}$ and $\mathrm{WG}_{2}, D_{1}$ is the diameter of $\mathrm{WG}_{1}$, and $k$ is the wake decay constant. $A_{1}$ presents the shadowed area by $\mathrm{WG}_{1}$ at the position of $\mathrm{WG}_{2}$, and $A_{1 \rightarrow 2}^{\text {overlap }}$ is the overlap area between $A_{1}$ and the swept area of $\mathrm{WG}_{2}$. In the Park model, the arriving wind speed of the $i$-th $\mathrm{WG}, V_{i}$, can be calculated using the following equation,

$$
V_{i}=U_{\infty}\left[1-2 \sqrt{\sum_{\substack{j=1 \\ j \neq i}}^{n}\left\{\alpha_{j}\left(\frac{D_{j}}{D_{j}+2 k x_{j i}}\right)^{2} \frac{A_{j \rightarrow i}^{\text {overlap }}}{A_{i}}\right\}^{2}}\right]
$$

where $n$ is the total number of WGs, and $\alpha_{j}$ the axial induction factor of the upstream $\mathrm{WG}_{j}$.

\section{Case Studies}

As a disturbance, SG5 in the model system is assumed to be tripped at $t=300 \mathrm{~s}$; it supplies $70 \mathrm{MW}$ to the load prior to a disturbance. The reserve of the WPP is assumed to be $10 \%$ of the available power before the disturbance. This 
study considers three cases with a wind direction of $0^{\circ}$ or $90^{\circ}$ and a wind speed of $10 \mathrm{~m} / \mathrm{s}$ or $11 \mathrm{~m} / \mathrm{s}$. Table 1 shows the arriving wind speeds of PMSGs for three cases. Each cell in Table 1 represents a PMSG in a model system and the value at each cell indicates the arriving wind speed of each PMSG calculated by using (13). In Cases 1 and 2, the wind direction is $0^{\circ}$ and thus five PMSGs in the same column have the same arriving wind speed. In Case 3 , the wind direction is changed to $90^{\circ}$ and thus four PMSGs in the same row have the same arriving wind speed. The wind direction is shown in Fig. 3. The performance of the optimal reserve allocation is compared to those of the dynamic reserve allocation in [12] and MPPT operation.

\subsection{Case1: wind speed of $10 \mathrm{~m} / \mathrm{s}$, wind direction of $0^{\circ}$}

Fig. 8 shows the simulation results for Case 1. As shown in Fig. 8(b), the WPP output in MPPT operation is $41 \mathrm{MW}$ and those of both reserve allocations are $37 \mathrm{MW}$ until $300 \mathrm{~s}$, which indicates that the WPP retains the reserve of $4 \mathrm{MW}$ before the disturbance. After the disturbance, the reserve and $\mathrm{KE}$ are released because of the change in power reference from $P_{W G, i}^{c m d}$ to $P_{M P P T}$. The optimal allocation releases a 33 MWs larger amount of KE than the dynamic allocation during the period of time from $300 \mathrm{~s}$ to $315 \mathrm{~s}$. Thus, the frequency nadir for the optimal allocation is $59.617 \mathrm{~Hz}$, which is $0.040 \mathrm{~Hz}$ higher than that of the dynamic allocation, and higher than that of MPPT

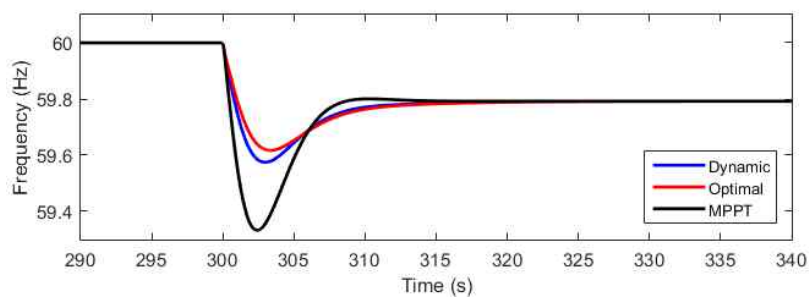

(a) System frequencies

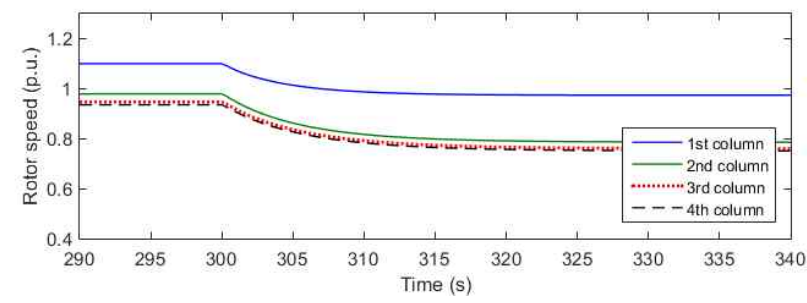

(c) Rotor speeds for a dynamic reserve allocation

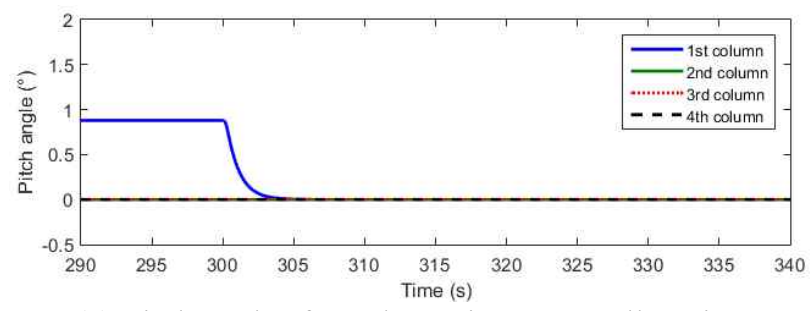

(e) Pitch angles for a dynamic reserve allocation operation by $0.284 \mathrm{~Hz}$, as shown in Fig. 8(a)

As shown in Figs. 8(c) and 8(d), prior to the disturbance, the rotor speeds for the optimal allocation are 1.100 p.u. for PMSGs in the first column, $1.012 \mathrm{p} . \mathrm{u}$. for the second column, 0.996 p.u. for the third column, and 0.991 p.u. for the fourth column; the rotor speeds for the dynamic allocation are 1.100 p.u., 0.979 p.u., 0.947 p.u., and 0.935 p.u., respectively. The total released KE for the optimal and dynamic allocation is $526 \mathrm{MWs}$ and $493 \mathrm{MWs,}$ respectively.

As shown in Figs. 8(e) and 8(f), prior to the disturbance, the pitch angles for the optimal allocation are zero, whereas the pitch angle of the first columns in the dynamic allocation is $0.8^{\circ}$. This is because pitching is used to obtain the reserve in the dynamic allocation.

\subsection{Case 2: wind speed of $11 \mathrm{~m} / \mathrm{s}$, wind direction of $0^{\circ}$}

Fig. 9 shows the results for Case 2, which is identical to Case 1 except for the wind speed. The optimal allocation releases a 41 MWs larger amount of KE than the dynamic allocation from $300 \mathrm{~s}$ to $315 \mathrm{~s}$. The frequency nadir for the optimal allocation is $59.686 \mathrm{~Hz}$, which is $0.050 \mathrm{~Hz}$ higher than that for the dynamic allocation, and higher than that of MPPT operation by $0.314 \mathrm{~Hz}$.

Prior to the disturbance, the rotor speeds of PMSGs for the optimal allocation reaches 1.100 p.u., which is the maximum value, whereas the rotor speeds in the first, second,

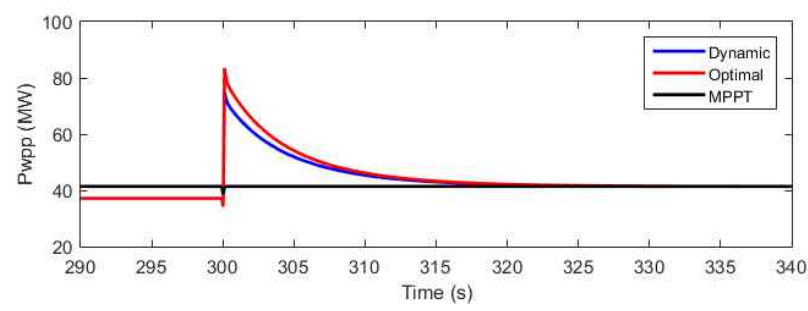

(b) Active power outputs of the WPP

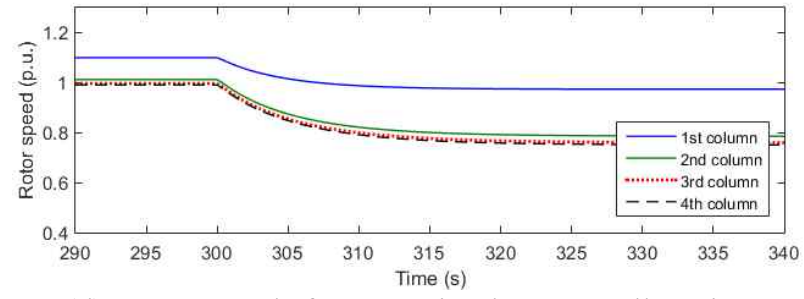

(d) Rotor speeds for an optimal reserve allocation

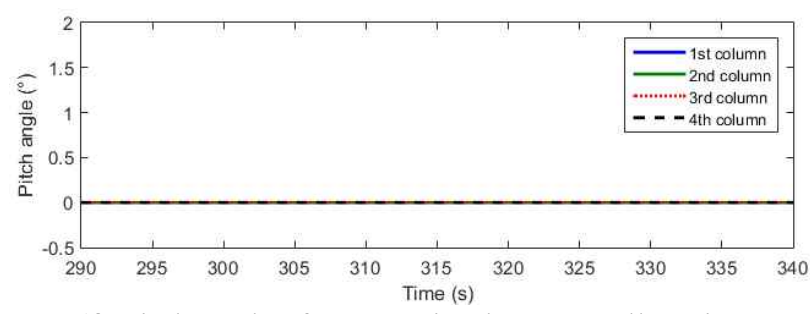

(f) Pitch angles for an optimal reserve allocation

Fig. 8. Results for Case 1 


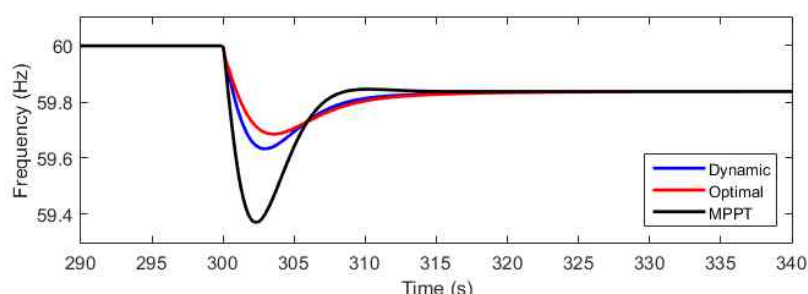

(a) System frequencies

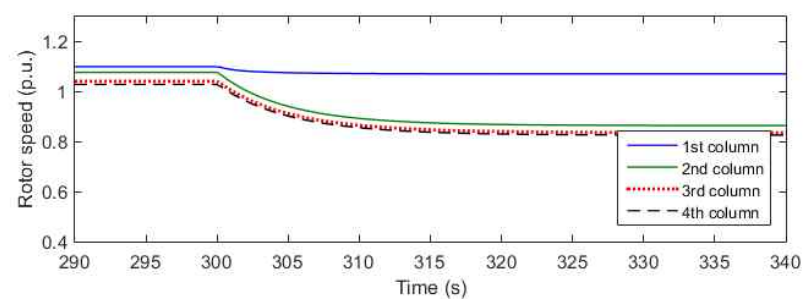

(c) Rotor speeds for a dynamic reserve allocation

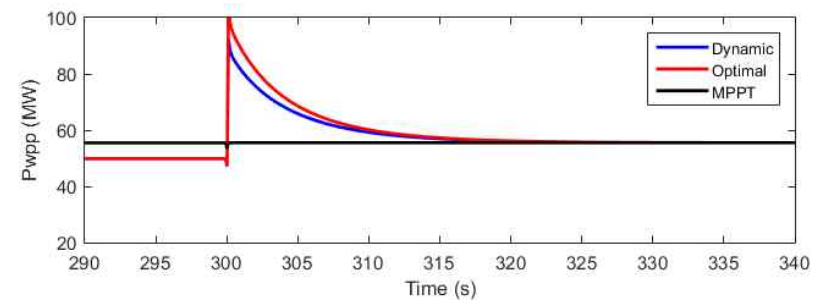

(b) Active power outputs of the WPP

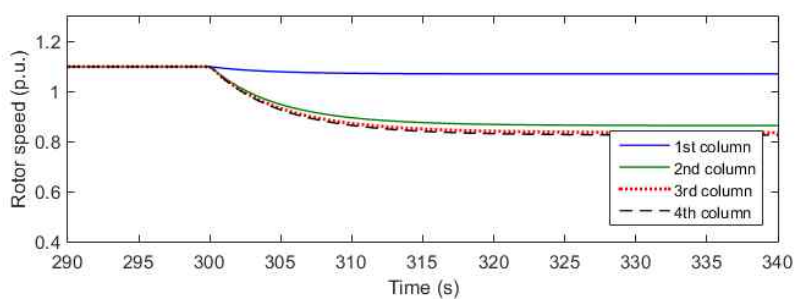

(d) Rotor speeds for an optimal reserve allocation

Fig. 9. Results for Case 2

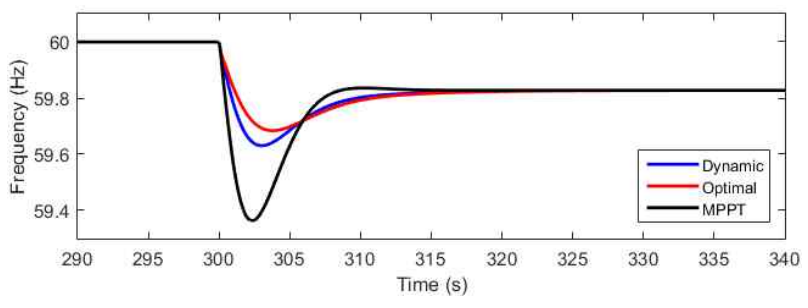

(a) System frequencies

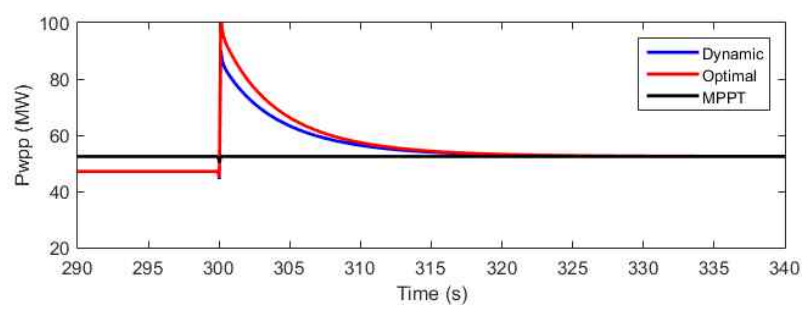

(b) Active power outputs of the WPP

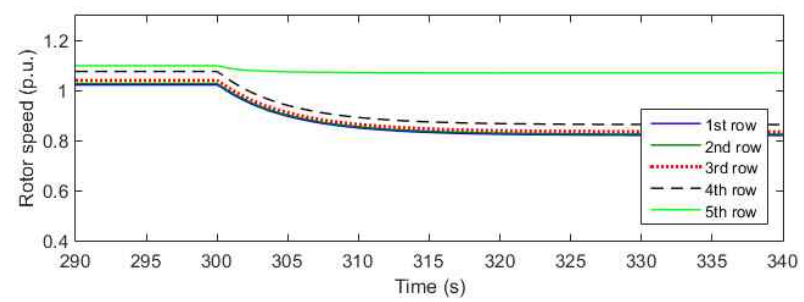

(c) Rotor speeds for a dynamic reserve allocation

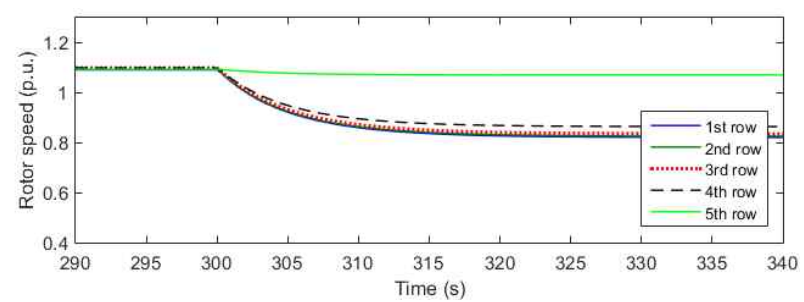

(d) Rotor speeds for an optimal reserve allocation

Fig. 10. Results for Case 3 third, and fourth columns for the dynamic allocation are 1.100 p.u., 1.080 p.u., 1.042 p.u., and 1.030 p.u., respectively. The total released $\mathrm{KE}$ for the optimal and dynamic allocation are $605 \mathrm{MWs}$ and $564 \mathrm{MWs}$, respectively. Thus, the optimal allocation releases a larger amount of KE than the dynamic allocation.

\subsection{Case 3: wind speed of $11 \mathrm{~m} / \mathrm{s}$, wind direction of $90^{\circ}$}

Fig. 10 shows the results for Case 3 , which is identical to Case 2 except for the wind direction. The optimal allocation releases a $46 \mathrm{MWs}$ larger amount of KE than the dynamic allocation. The frequency nadir for the optimal allocation is $59.684 \mathrm{~Hz}$, which is $0.050 \mathrm{~Hz}$ higher than that for the dynamic allocation, and higher than that of MPPT operation by $0.320 \mathrm{~Hz}$.

Prior to the disturbance, the rotor speeds of PMSGs for the optimal allocation reaches 1.100 p.u., while the rotor speeds of the first, second, third, fourth, and fifth rows for the dynamic allocation are 1.100 p.u., 1.080 p.u., 1.040 p.u., 1.030 p.u., and 1.020 p.u., respectively. The total released $\mathrm{KE}$ for the optimal and dynamic allocation is $602 \mathrm{MWs}$ and $556 \mathrm{MWs}$, respectively.

The results of the above three cases demonstrate that the proposed optimal allocation improves the frequency nadir more than the dynamic reserve allocation by releasing a larger amount of KE stored in the WPP under the various wind conditions.

\section{Conclusions}

This paper proposes an optimization formulation that allocates the required reserve to PMSGs to maximize the KE stored in the WPP. The proposed optimization 
formulation allows a PMSG with a smaller rotor speed to retain sufficient $\mathrm{KE}$ to maximize the amount of stored $\mathrm{KE}$ in the WPP.

The results indicate that the proposed optimal reserve allocation retains a larger amount of KE with the same reserve and successfully increases the frequency nadir by releasing the stored KE in a WPP in the case of a disturbance. The proposed optimal reserve allocation shows better performance than the conventional dynamic reserve allocation under various wind conditions.

\section{Acknowledgements}

This work was supported partly by the National Research Foundation of Korea (NRF) grant funded by the Korea government (MSIP) (No.2010-0028509) and partly by the New \& Renewable Energy of the Korea Institute of Energy Technology Evaluation and Planning (KETEP) grant funded by the Korea government Ministry of Trade, industry \& Energy (No. 2011T100200064).

\section{References}

[1] Global Wind Energy Council, "Global wind report: annual market update 2013," Apr. 2014.

[2] The Ministry of Knowledge Economy, "The 6th basic plan of long term electricity supply and demand," Feb. 2013.

[3] O. Anaya-lara, N. Jenkins, J. Ekanayake, P. Cartwright, and M. Hughes, Wind Energy Generation Modeling and Control: John Wiley \& Sons, Ltd, 2009.

[4] Hydro Québec, "Transmission provider technical requirements for the connection of power plants to the Hydro Québec transmission system." Feb. 2009.

[5] EirGrid, "EirGrid Grid Code Version 5.0", Oct. 2013.

[6] J. Ekanayake and N. Jenkins, "Comparison of the response of doubly fed and fixed-speed induction generator wind turbines to changes in network frequency," IEEE Trans. Energy Convers., vol. 19, no. 4, pp. 800-802, Dec. 2004.

[7] E. Loukarakis, I. Margaris, and P. Moutis, "Frequency control support and participation methods provided by wind generation," in Proceedings of IEEE Electrical Power \& Energy Conference, Montreal, Canada, Oct. 2009.

[8] E. Muljadi, V. Gevorgian, and M. Singh, "Understanding inertial and frequency response of wind power plants," in Proceedings of IEEE Symposium on Power Electronics and Machines in Wind Applications, Denver, USA, Jul. 2012.

[9] R. Sakamoto, T. Senjyu, N. Urasaki, T. Funabashi, and H. Fujita, "Output power leveling of wind turbine generators using pitch angle control for all operating regions in wind farm," in Proceedings of Intelligent
Systems Application to Power Systems, Arlington, USA, Nov. 2005.

[10] R. de Almeida, E. Castronuovo, and J. Lopes, "Optimum generation control in wind parks when carrying out system operator requests," IEEE Trans. Power Syst., vol. 21, no. 2, pp. 718-725, May 2006.

[11] A. Žertek, G. Verbič, and M. Pantoš, “Optimised control approach for frequency control contribution of variable speed wind turbines," IET Renew. Power Gener., vol. 6, no. 1, pp. 17-23, Jan. 2012.

[12] L.-R. Chang-Chien, C.-M. Hung, and Y.-C. Yin, "Dynamic reserve allocation for system contingency by DFIG wind farms," IEEE Trans. Power Syst., vol. 23, no. 2, pp. 729-736, May 2008.

[13] P. Boggs and J. Tolle, "Sequential quadratic programming," Acta Numerica, vol. 4, pp. 1-51, 1996.

[14] I. Katic, J. Højstrup, and N. O. Jensen, "A simple model for cluster efficiency," in Proceedings of European Wind Energy Association Conference and Exhibition, Rome, Italy, Oct. 1986.

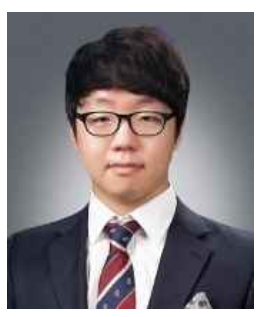

Gihwan Yoon He received his B.S. degree from Chonbuk National University, Korea in 2013. He is currently pursuing an M.S. degree at Chonbuk National University. $\mathrm{He}$ is also an assistant researcher at the Wind Energy Grid-adaptive Technology (WeGAT) Research Center, which is supported by the Ministry of Science, ICT, and Future Planning (MSIP), Korea. His research interest is the development of wind energy grid integration techniques.

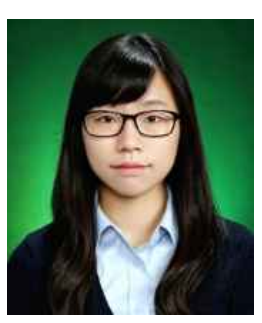

Hyewon Lee She received the B.S. degree from Chonbuk National University, Korea in 2010. She is studying for her Ph.D. degree at Chonbuk National University and is also an assistant researcher at the WeGAT Research Center supported by the MSIP, Korea. Her research interest is the development of wind energy grid integration techniques.

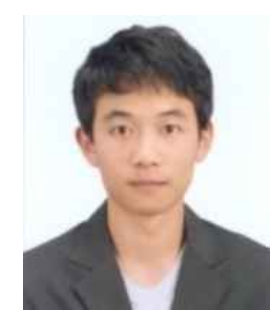

Jinsik Lee He received his B.S. and M.S. degrees from Chonbuk National Uni-versity, Korea in 2011 and 2013. $\mathrm{He}$ is currently pursuing his $\mathrm{Ph}$. D. degree at Chonbuk National University. $\mathrm{He}$ is also an assistant researcher at the WeGAT Research Center. His research interests are plant control systems for wind power plants. 


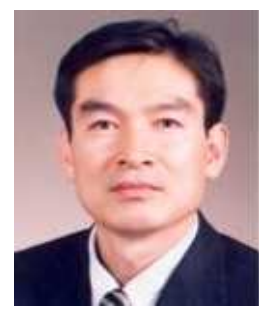

Gi-Gab Yoon $\mathrm{He}$ received his B.S., M.S., and Ph.D. degree in Electrical Engineering from Hanyang University, Seoul, Korea, in 1983, 1988, and 1999. $\mathrm{He}$ has over 25 years of research experience in the field of power systems. $\mathrm{He}$ is presently principal research engineer in Offshore Wind R\&BD

Center at KEPRI (Korea Electric Power Research Institute). His research field includes offshore wind power generation system, distributed generation system and control of power system.

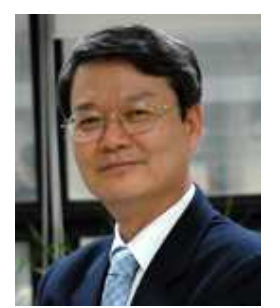

Jong Keun Park He was born in Republic of Korea in 1952. He received B.S. degree in electrical engineering from Seoul National University, Korea in 1973 and his M.S. and Ph.D. degrees in electrical engineering from The University of Tokyo, Japan in 1979 and 1982, respectively. He worked as a Researcher at the Toshiba Heavy Apparatus Laboratory in 1982. He was a Visiting Professor with the Technology and Policy Program and Laboratory for Electromagnetic and Electronic Systems, Massachusetts Institute of Technology, Cambridge, in 1992. He is currently a Professor in the School of Electrical Engineering, Seoul National University. $\mathrm{He}$ is a fellow of the Institute of Electrical and Electronics Engineers (IEEE) and the Institution of Electrical Engineers (IEE). Also he was a President of Korean Institute of Electrical Engineers (KIEE) in 2010.

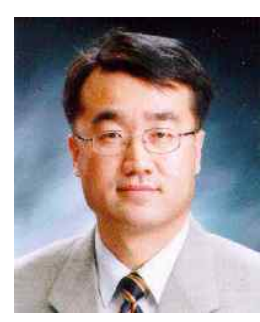

Yong Cheol Kang He received his B.S., M.S., and Ph.D. degrees from Seoul National University, Korea, in 1991, 1993, and 1997, respectively. $\mathrm{He}$ has been with Chonbuk National University, Korea, since 1999. He is currently a professor at Chonbuk National University, Korea, and the director of the WeGAT Research Center, supported by the MSIP, Korea. He is also with the Smart Grid Research Center at Chonbuk National University. His research interests are the development of new protection and control systems for wind power plants and the enhancement of wind energy penetration levels. 